Revista de Psicología Vol. 32 (2), 2014 (ISSN 0254-9247)

\title{
Estrategias de afrontamiento y ansiedad ante exámenes en estudiantes universitarios
}

\author{
Leandro Martín Casari ${ }^{1}$, Johana Anglada ${ }^{2}$ y Celeste Daher ${ }^{3}$ \\ Pontificia Universidad Católica Argentina, ${ }^{1}$ \\ Universidad del Aconcagua ${ }^{2,3}$ y CONICET CCT ${ }^{1,3}$
}

\begin{abstract}
Este artículo analizó las estrategias de afrontamiento y la ansiedad ante situación de examen en 140 estudiantes universitarios de Psicología de la Universidad del Aconcagua de la provincia de Mendoza. Además, evaluó las diferencias y el grado de relación entre estas dos variables en cuanto a género, materias rendidas y año académico. Los resultados mostraron que las estrategias de afrontamiento fueron la búsqueda de Gratificaciones Alternativas y la Descarga Emocional; además, que los estudiantes presentan un nivel bajo de ansiedad. Se encontraron relaciones significativas entre ansiedad ante exámenes y estrategias de afrontamiento, como también entre estas variables en relación al género y el año de cursado. No se encontraron resultados significativos al correlacionar con cantidad de materias rendidas.

Palabras clave: ansiedad ante exámenes, estrategias de afrontamiento, estudiantes universitarios
\end{abstract}

\section{Coping strategies and exam anxiety in college students}

This paper analyzed the coping strategies and exam anxiety in 140 Psychology majors at the University of the Aconcagua, in the state of Mendoza. It also evaluated the differences and the relationship between these two variables according to gender, completed courses and academic year. Results showed that the coping strategies included the Search for Alternative

1 Licenciado en Psicología por Universidad Nacional de San Luis, Argentina y docente de Trabajo de Integración Final en Pontificia Universidad Católica Argentina, Sede Mendoza. Dirección postal: INCIHUSA - CONICET CCT Mendoza. Avenida Ruiz Leal s/n, Parque General San Martín (5700), Mendoza, Argentina. Contacto: leandromartincasari@gmail.com

2 Licenciada en Psicología por la Universidad del Aconcagua, Mendoza, Argentina y especialista en terapia cognitivo conductual. Dirección postal: Barrio Jardín Aeroparque M10 C27. Las Heras (5539), Mendoza, Argentina. Contacto: johaanglada@hotmail.com

3 Licenciada en Psicología por la Universidad Nacional de San Luis, Argentina y docente de Psicología del Desarrollo 1 en la Facultad de Psicología de Universidad del Aconcagua, Mendoza, Argentina. Dirección postal: INCIHUSA - CONICET CCT Mendoza. Avenida Ruiz Leal s/n, Parque General San Martín (5700), Mendoza, Argentina. Contacto: celestedaher@gmail.com 
Bonuses and Emotional Discharge; students also reported a low level of anxiety. Significant associations were found between these two variables, as well in relation to gender and the academic year. No significant results were found in the relationship with completed courses. Key words: exams anxiety, coping strategies, college students 
Se considera al ámbito académico como un conjunto de situaciones estresantes por las cuales las personas experimentan falta de control sobre la situación y así generan respuestas de estrés y fracaso académico, las cuales pueden afectar tanto a profesores como a estudiantes. A estos individuos, el ámbito académico le plantea problemáticas generadoras de estrés que deben enfrentar con los recursos que tienen (García-Ros, Pérez-González, Pérez-Blasco \& Natividad, 2012; Piemontesi \& Heredia 2009).

Considerando que la respuesta al estrés depende de la disposición personal para hacer frente a las demandas del contexto como a la propia situación o evento, se puede decir que no todos los estudiantes padecen estrés ante las mismas circunstancias, como pueden ser, la falta de tiempo para poder cumplir con las actividades académicas, la sobrecarga académica y la realización de un examen. Por lo tanto, no es la situación de examen la que genera estrés, sino que el mismo se vincula con el "agobio" que provoca a los estudiantes la relación entre la cantidad trabajo/tiempo, es decir, al percibir la cantidad de tareas por hacer y el poco tiempo para llevarlas a cabo (Polo, Hernández López \& Pozo Muñoz, 1996).

De igual manera, podría afirmarse que hay estresores presentes en una carrera universitaria, por lo que la situación de examen constituye una de las principales fuentes de estrés y tensión para los estudiantes universitarios. Asimismo, en cada caso particular, el nivel de ansiedad y el modo de enfrentar la situación de examen están íntimamente relacionados (Montaña, 2011). Así también, es importante resaltar que el estrés ante un examen tiene consecuencias en el plano conductual, cognitivo y fisiológico con efectos a corto y largo plazo particulares en cada estudiante (Martín Monzón, 2007).

A su vez, existe un nivel de ansiedad útil, funcional o necesaria que moviliza a actuar, pero cuando la ansiedad supera ciertos límites, 
puede ocasionar consecuencias desadaptativas. Esto puede suceder en situaciones de examen y, por lo tanto, llevar al estudiante a un menor rendimiento en su desempeño (Castellanos Cárdenas, Guarnizo Castillo \& Salamanca Camargo, 2011).

Entrando al tema del presente estudio, el afrontamiento ante exámenes podría definirse como un proceso de transacción entre la persona y el ambiente, con el uso flexible y combinado de distintas estrategias, que no son uniformemente efectivas, sino que los resultados son multidireccionales dependiendo de diversos factores. Es así que las estrategias de afrontamiento efectivas en la situación de examen pueden no serlo en otras situaciones cotidianas. Un dato interesante a tener en cuenta es que las estrategias de afrontamiento no deberían ser llamadas adaptativas o desadaptativas, sino que serían más bien funcionales y particulares de cada persona, es decir, es necesario considerar para quién, bajo qué circunstancias y ante qué situación esa estrategia tiene consecuencias adaptativas (Piemontesi \& Heredia, 2009).

Con respecto, a la ansiedad ante los exámenes es importante considerar la definición de Hodapp (Hodapp, Glanzmann \& Laux, 1995 citado en Heredia, Piemontesi, Furlan \& Volker Hodapp, 2008), quien la entiende como un rasgo especifico-situacional, que se caracteriza por la predisposición del estudiante a actuar con elevada ansiedad en los ambientes relacionados con el rendimiento. Gutiérrez Calvo y Avero (1995) agregan que la ansiedad ante los exámenes es una tendencia relativamente estable en la mayoría de los estudiantes a responder con altos niveles de ansiedad en situaciones en las que están siendo evaluados, presentando como aspecto central la preocupación por su posible mal desempeńo en el examen y las consecuencias para su autoestima, estatus, etc.

En la presente investigación se empleará el Modelo de la Reducción de la Eficiencia, desarrollado por Gutiérrez Calvo y Avero (1995), el cual postula que la preocupación, componente cognitivo de la ansiedad, se da sobre un mecanismo auto-regulatorio (implicado en las actividades meta-cognitivas durante el aprendizaje y rendimiento). Dicho componente surge cuando el estudiante compara los conocimientos que tiene 
con los realmente necesarios para conseguir aprobar el examen. De esta manera, la preocupación aumentará cuando los conocimientos que el alumno tenga no alcancen estos estándares necesarios, lo que producirá que este mecanismo auto-regulatorio aumente los recursos del estudiante para disminuir la preocupación y obtener un buen rendimiento en el examen. Estos recursos extras que utilizan las estudiantes con elevada ansiedad explican por qué hay sujetos con altos niveles de ansiedad pero buen rendimiento, a diferencia de los estudiantes con bajos niveles ansiedad, cuyos esfuerzos son mayores para tener un buen rendimiento al procesar de manera menos eficiente. De este modo, como plantean algunos autores, puede esperarse que el estudiante al preparar un examen aumente su preocupación e implemente recursos disponibles en la memoria operativa, como por ejemplo dedicar más tiempo de estudio y de repaso, emplear alternativas de procesamiento cognitivo, planificar las actividades, buscar información que ayude a lograr una mejor preparación, estudiar con un compañero/a, entre otras. Todas ellas con el objetivo de disminuir la preocupación evitando las posibilidades de fracaso (Piemontesi \& Heredia, 2009).

Para conocer la percepción que los estudiantes universitarios tienen de la situación de evaluación, resulta interesante tener en cuenta un reciente estudio de casos de carácter cualitativo, realizado por Ricoy y Fernandez-Rodriguez (2013). Los resultados ponen de manifiesto que ante un examen predominan las sensaciones negativas (especialmente nerviosismo) por sobre las positivas, además los autores resaltan que la mayoría de los estudiantes reconocen que dedican poco tiempo a programar la materia y al estudio, lo que hace que utilicen estrategias cognitivas para memorizar la información y los contenidos de la materia al no tener el tiempo necesario para reflexionarlo. Ello a su vez genera sensaciones negativas que se manifiestan de forma perjudicial sobre su calidad de vida.

El conocimiento sobre la predominancia de las percepciones negativas que tienen los estudiantes universitarios de un examen, el cual además comprende algunos elementos de los principales estresores tales como la preparación para impedir el evento, la confrontación al 
estresor, la incertidumbre de los resultados del examen y el afrontamiento ante las consecuencias de estos resultados, ha permitido ampliar en los últimos años el interés acerca de cómo los estudiantes enfrentan su experiencia de ansiedad y cuáles son las estrategias de afrontamiento implementadas por estos para afrontar la situación estresante de examen. Asimismo se ha hecho foco en el rendimiento académico y la presencia de síntomas o quejas somáticas experimentadas por los estudiantes en épocas de examen, así como en sus posibles relaciones con estrategias de afrontamiento y los niveles de ansiedad (Piemontesi, Heredia, Furlan, Sánchez-Rosas \& Martínez, 2012).

También se ha estudiado el nivel de estrés en relación a los exámenes y al período próximo a sus fechas, además de analizar la influencia de determinados indicadores de salud y del autoconcepto académico. En general, los resultados muestran un aumento en el nivel de estrés de los universitarios durante el período de exámenes. Del mismo modo, se han hallado efectos sobre la salud (ansiedad, consumo de tabaco, cafeína o fármacos, alteraciones en el sueño y en la ingesta de alimentos) y sobre el autoconcepto académico de los estudiantes (peor nivel de autoconcepto académico) durante el período de presencia del estresor (Martín Monzón, 2007).

Rusińol, Gomez y Valdés en el año 1993 evaluaron el afrontamiento y el nivel de ansiedad en 256 alumnos de $2^{\circ}$ año de la Facultad de Medicina y Psicología de la Universidad de Barcelona. Los principales resultados fueron que los estudiantes de medicina presentan mayor reevaluación positiva; mientras que en las mujeres la ansiedad correlaciona con las escalas "distanciamiento", "aceptación de la responsabilidad" y "reevaluación positiva", en los hombres la ansiedad correlaciona con "distanciamiento".

Cabe aclarar que, si bien existe diversidad de estudios que evalúan las estrategias de afrontamiento y ansiedad en situación de examen a estudiantes universitarios, se han utilizado también variedad de instrumentos, que modifican los resultados de una investigación a otra.

Montańa (2011) propuso evaluar las estrategias de afrontamiento y ansiedad ante situación de examen en estudiantes universitarios de 
Psicología. Los resultados arrojaron que a mayores niveles de ansiedad, más se utiliza la estrategia de distanciamiento y de escape-evitación, y menos la resolución de problemas. En relación al género las mujeres utilizan las estrategias de Resolución de Problemas y Búsqueda de Apoyo Social; mientras que los hombres no utilizan una estrategia como preferencia, pero sí presentan mayores niveles de emocionalidad, preocupación e interferencias. En cuanto a los niveles de ansiedad en relación a las diferencias de género, los resultados arrojaron que en los varones a mayores niveles de ansiedad asociado al uso de autocontrol, hay una menor utilización de Resolución de Problema y Distanciamiento, en cambio en las mujeres a mayores niveles de ansiedad, más utilizan la estrategia de confrontación. En relación al año académico hay diferencias entre estudiantes de $1^{\circ}$ año y $5^{\circ}$ año; mientras que los primeros no utilizan una estrategia como preferencia, los alumnos de $5^{\circ}$ año recurren a la Resolución de Problemas con mayores niveles de emocionalidad, preocupación y falta de confianza.

Un estudio realizado por Furlan, Sánchez Rosas, Heredia, Piemontesi, Illbele y Martínez (2012) se propuso analizar las relaciones entre ansiedad frente a los exámenes, las estrategias de aprendizaje y los estilos de afrontamiento en estudiantes universitarios ante el estrés académico con baja, moderada y alta ansiedad en los exámenes. Los resultados permiten afirmar que se deben tener en cuenta las estrategias de aprendizaje y de afrontamiento al analizar la ansiedad frente a los exámenes. En esta sentido, se encontró que los alumnos con elevada ansiedad utilizan con mayor frecuencia las estrategias superficiales de procesamiento con estilos de afrontamiento con actividad mental autocentrada o recurrente en torno al problema; mientras que los estudiantes con baja ansiedad utilizan estrategias reflexivas y críticas, aceptan la situaciones problemáticas, reevaluándolas positivamente y actuando para transformarlas. Asimismo, descubrieron que cada dimensión de la ansiedad se relaciona de manera particular con las estrategias de aprendizaje y los estilos de afrontamiento. Es así como la preocupación se correlaciona en forma positiva con las estrategias de repetición y regulación del esfuerzo, y con estilos más o menos adaptativos de afrontamiento, mientras que la falta 
de confianza y la interferencia se relacionan negativamente con estrategias de aprendizaje reflexivo y crítico, y positivamente con los estilos menos eficaces para afrontar el estrés.

Tal como lo plantean Piemontesi y colaboradores (2012), las investigaciones que evalúan las relaciones entre la ansiedad ante los exámenes y las estrategias de afrontamiento han arrojado resultados dispares y, en cierto modo, contradictorios. Los trabajos más dispares son los que han implementado instrumentos unidimensionales de la ansiedad ante el examen, los cuales sugieren que una ansiedad elevada en los estudiantes se relaciona con estrategias consideradas desadaptativas como a estrategias adaptativas.

Siguiendo en la línea de la relación entre ansiedad y rendimiento académico, Álvarez, Aguilar y Lorenzo (2012) se propusieron determinar en qué medida hay manifestaciones de síntomas de ansiedad ante los exámenes y qué relación guardan con el rendimiento académico. Se concluyó que una gran población de estudiantes universitarios manifiesta una ansiedad preocupante o muy preocupante frente a los exámenes en respuestas cognitivas, fisiológicas y motoras, sin embargo el rendimiento académico no presentó relación con estos niveles de ansiedad. Similares resultados fueron postulados por Hernández (2005), quien en un estudio realizado con una muestra de 28.559 alumnos de 16 universidades espańolas, encontró que un $21 \%$ de los evaluados manifestaba tener una alta ansiedad para enfrentar un examen, por lo que podrían necesitar ayuda especializada.

Ávila-Toscano, Hoyos Pacheco, González y Cabrales Polo (2011) evaluaron la ansiedad ante exámenes, los tipos de pruebas académicas y el rendimiento académico en estudiantes universitarios. Los resultados mostraron que las manifestaciones cognitivas son las más prevalentes aunque las menos intensas; sin embargo, se identificaron relaciones significativas entre las tres manifestaciones, logrando correlaciones positivas entre cada uno de los conjuntos sintomáticos valorados. Es decir que, a medida que aumenta la tensión cognitiva, crece la ansiedad motora y fisiológica. Además, pudieron observar que la aparición de preocupaciones es el síntoma identificado con mayor frecuencia entre 
los universitarios $(76.5 \%)$, seguido en orden de aparición por miedo (47\%), así como por el desarrollo de pensamientos y sentimientos negativos. Los síntomas cognitivos de ansiedad se presentan de forma significativa ante la realización de exámenes orales de tipo individual.

De igual manera, Sicre (2013) evaluó las estrategias de afrontamiento en estudiantes universitarios ante el estrés académico en situación de examen. Los resultados revelaron que la estrategia de afrontamiento más utilizada por los estudiantes es Afrontamiento por Aproximación Cognitiva, teniendo un nivel de estrés prioritariamente moderado. El autor también señala que los síntomas del estrés académico se presentan solo algunas veces en los alumnos, es decir, que no ante todas las situaciones de examen los alumnos muestran estrés. Allí no se encontraron correlaciones significativas entre las variables en estudio, pero sí se presentaron correlaciones entre la cantidad de materias rendidas y las estrategias de afrontamiento utilizadas: a mayor número de asignaturas alcanzadas, mayores valores en Afrontamiento por Aproximación Cognitiva y Conductual.

Acerca de la existencias de diferencias significativas según género en el afrontamiento ante situaciones académicas estresantes, se encontró que los hombres utilizan con mayor frecuencia las estrategias de Reevaluación Positiva y Planificación, mientras que las mujeres optan principalmente por la Búsqueda de Apoyo (Cabanach, Fariña, Freire, González \& Ferradás, 2013).

Por estos motivos resultó importante investigar esta temática en los estudiantes universitarios de psicología, con el objetivo de saber y comprender la manera en que estos enfrentan la situación de examen y el manejo de la ansiedad ante la misma. De esta manera, se pretende aportar herramientas útiles para que los estudiantes enfrenten la situación de examen con estrategias adaptativas y con un nivel funcional de ansiedad que no dificulte el rendimiento en el examen. Todo ello permitirá no solo detectar sino también prevenir el fracaso, el estrés académico y los aplazos de los alumnos. Comprendiendo esta última como la principal justificación del presente estudio. 
En este sentido, se tienen como objetivos específicos: (a) describir el nivel de ansiedad y estrategias de afrontamiento más utilizadas en estudiantes universitarios; (b) evaluar la existencia de correlaciones significativas entre estrategias de afrontamiento y ansiedad antes los exámenes en estudiantes universitarios de psicología; y (c) analizar la presencia de correlaciones y diferencias significativas entre estrategias de afrontamiento y niveles de ansiedad ante exámenes, con relación al género, año académico y cantidad de asignaturas rendidas.

\section{Método}

\section{Participantes}

La muestra estuvo conformada por 140 estudiantes ( $82 \%$ mujeres y $18 \%$ hombres) que cursaban de $1^{\circ}$ a $5^{\circ}$ año del pregrado en psicología ( $14 \%$ primer año, $16 \%$ segundo ańo, $18 \%$ tercer año, $26 \%$ cuarto año y $26 \%$ quinto año), en la Universidad del Aconcagua, cuya edad se encontraba en un rango entre 18 y 40 años $\left(M_{\text {(edad) }}=23.07, D E_{\text {(edad) }}=3.394\right)$. A su vez, se registró que los estudiantes habían rendido entre 2 y 46 materias $\left(M_{\text {(cursos rendidos) }}=25, D E_{\text {(cursos rendidos) }}=15\right)$.

\section{Medidas e instrumentos de medición}

1. Inventario de Respuestas de Afrontamiento (CRI Adult - Form, Coping Responses Inventory) de Rudolph Moos, elaborado (1993) y adaptado por Mikulic y Crespi (2008). Es un inventario autoadministrable que evalúa las respuestas de afrontamiento de una persona ante una situación estresante. Consta de 48 preguntas en una escala Likert de 0 a 3: 'Nunca', 'Raras veces', 'Algunas veces' y, 'Muy a menudo'. Las categorías de evaluación se dividen en Análisis Lógico, Reformulación Positiva, Búsqueda de Apoyo, Solución de Problemas, Evitación Cognitiva, AceptaciónResignación, Búsqueda de gratificaciones alternativas y Descarga Emocional. Para la corrección, los puntajes brutos obtenidos en 
Estrategias de afrontamiento y ansiedad ante exámenes en estudiantes universitarios / Casari et al.

cada categoría se transformaron en percentiles. En cuanto a su confiablidad, la escala presenta valores aceptables en el alfa de Cronbach (superiores a .75) en la escala total y en las subescalas, además el análisis factorial arrojó dos factores principales coincidiendo con los postulados iniciales del autor.

2. S-CTAS, Escala de Ansiedad Cognitiva frente a los exámenes, elaborada por Cassady y Johnson en 2002, y adaptada por Furlan, Perez, Moyano y Cassady (2010). Esta escala es un autoinforme de 16 ítems que mide manifestaciones cognitivas de ansiedad frente a los exámenes en estudiantes universitarios. Posee ítems que indican niveles bajos y altos de ansiedad, permitiendo clasificar a los estudiantes según su nivel de ansiedad alto, moderado y bajo. Sus ítems se refieren a la tendencia a preocuparse por un eventual mal rendimiento, poseer pensamientos irrelevantes en cuanto a la tarea durante el examen y en los períodos de preparación del mismo. La escala posee formato de respuesta tipo Likert con 4 opciones posibles, desde "Nada frecuente en mí" hasta "Muy frecuente en mí". El puntaje total es traducido a puntaje percentilar según los valores de referencia. Puntuaciones superiores a la media indican una elevada probabilidad de presentar dificultades emocionales asociadas a las evaluaciones, es decir, que presentan una alta ansiedad en situación de examen. En relación a los datos de confiabilidad y validez, los análisis factoriales de tipo exploratorio y confirmatorio realizados sugieren una estructura unidimensional de 16 ítems, con elevada consistencia interna y aceptable estabilidad temporal. Las evidencias de validez basada en criterio, respecto a rendimiento académico, perfeccionismo, autorregulación atencional y pensamientos automáticos negativos son teóricamente congruentes.

\section{Procedimiento y procesamiento de información}

Para dar inicio a la investigación se eligió una asignatura de cada año del pregrado en psicología, y se solicitó la autorización al profesor responsable para administrar los cuestionarios a los estudiantes de dicha materia, cuya colaboración fue voluntaria y anónima. El tiempo 
estimado para completar ambos cuestionarios fue de 15 minutos. Se explicó brevemente a los estudiantes en qué consistía la investigación y se les ofreció la posibilidad de una devolución del resultado a quien lo deseara. Al entregar ambos cuestionarios se les pidió que pensaran en una situación de examen reciente para completar cada técnica. Una vez recolectados los cuestionarios, se realizó un procesamiento de la información mediante estadística univariada a través del procesador estadístico SPSS 19.

\section{Resultados}

En función del primer objetivo planteado, los resultados de los niveles de ansiedad se presentan según género, ya que la escala utiliza puntajes distintos para cada sexo. Los resultados arrojaron que la media de los niveles de Ansiedad en las mujeres es $32 \pm 8$.5. Ello indica un Puntaje $T$ de 45, que se encuentra por debajo de la media (Furlan et. al., 2010), es decir, que las estudiantes mujeres presentan en general, una baja ansiedad ante los exámenes. Con respecto al sexo masculino, la media es de $34 \pm 8.2$. Esto equivale a un Puntaje $T$ de 50, que se encuentra por debajo de la media, lo cual implica que los estudiantes hombres presentan, en general, una baja ansiedad ante los exámenes. Por lo tanto, respondiendo al primer objetivo, tanto hombres como mujeres presentan un nivel bajo de ansiedad ante la situación de examen.

Por otro lado, las Estrategias de Afrontamiento que utilizan los estudiantes con mayor frecuencia ante un examen son evitativas y conductuales, tales como la búsqueda de gratificaciones alternativas y descarga emocional. La búsqueda de gratificaciones alternativas, arrojó un Puntaje $T$ de 62, lo que le corresponde un percentil de 84-93 y se clasifica como ampliamente por encima del promedio. Descarga Emocional obtuvo un Puntaje $T$ de 66 , con un percentil de $\geq 94$, que se clasifica como considerablemente por encima del promedio (Mikulic \& Crespi, 2008). Las demás estrategias de Afrontamiento puntuaron dentro del percentil 34-66, ubicándose dentro del promedio (ver Tabla 1). 


\section{Tabla 1}

Estrategias de Afrontamiento ante situación de examen $(n=140)$

\begin{tabular}{lccccc}
\hline $\begin{array}{l}\text { Estrategia de } \\
\text { Afrontamiento }\end{array}$ & $M$ & DE & Puntaje T & Percentil & Clasificación \\
\hline Análisis Lógico & 11.82 & 3.29 & 52 & $34-66$ & $\begin{array}{c}\text { Dentro del } \\
\text { promedio }\end{array}$ \\
$\begin{array}{l}\text { Reevaluación } \\
\text { Positiva }\end{array}$ & 12.53 & 2.52 & 53 & $34-66$ & $\begin{array}{c}\text { Dentro del } \\
\text { promedio }\end{array}$ \\
$\begin{array}{l}\text { Búsqueda de } \\
\text { Apoyo }\end{array}$ & 10.46 & 3.21 & 52 & $34-66$ & $\begin{array}{c}\text { Dentro del } \\
\text { promedio }\end{array}$ \\
$\begin{array}{l}\text { Solución de } \\
\text { Problemas }\end{array}$ & 12.59 & 3.03 & 52 & $34-66$ & $\begin{array}{c}\text { Dentro del } \\
\text { promedio }\end{array}$ \\
$\begin{array}{l}\text { Evitación } \\
\text { Cognitiva }\end{array}$ & 7.85 & 3.37 & 53 & $34-66$ & $\begin{array}{c}\text { Dentro del } \\
\text { promedio }\end{array}$ \\
$\begin{array}{l}\text { Aceptación- } \\
\text { Resignación }\end{array}$ & 5.81 & 3.63 & 47 & $34-66$ & $\begin{array}{c}\text { Dentro del } \\
\text { promedio }\end{array}$ \\
$\begin{array}{l}\text { Gratificaciones } \\
\text { Alternativas }\end{array}$ & 10.62 & 3.13 & 62 & $84-93$ & $\begin{array}{c}\text { Ampliamente } \\
\text { por encima del } \\
\text { promedio }\end{array}$ \\
$\begin{array}{l}\text { Descarga } \\
\text { Emocional }\end{array}$ & 8.69 & 2.88 & 66 & $\geq 94$ & $\begin{array}{c}\text { Considerablemente } \\
\text { por encima del } \\
\text { promedio }\end{array}$ \\
\hline
\end{tabular}

Seguidamente, se procedió a analizar si existían diferencias significativas entre estudiantes de distintos años académicos, a través del análisis de varianza unidireccional (ANOVA). Se encontraron múltiples diferencias significativas (ver Tabla 2). 


\section{Tabla 2}

ANOVA de ansiedad y afrontamiento entre estudiantes de distintos años académicos $(n=140)$

\begin{tabular}{|c|c|c|c|c|c|}
\hline & Año & $M$ & $D E$ & $F$ & $p$ \\
\hline \multirow{5}{*}{ Puntaje Ansiedad } & $1^{\circ}$ & 32.66 & 9.79 & \multirow{5}{*}{5.467} & \multirow{5}{*}{.000} \\
\hline & $2^{\circ}$ & 33.77 & 8.54 & & \\
\hline & $3^{\circ}$ & 38.66 & 6.09 & & \\
\hline & $4^{\circ}$ & 30.30 & 7.68 & & \\
\hline & $5^{\circ}$ & 29.78 & 7.88 & & \\
\hline \multirow{5}{*}{ Análisis Lógico } & $1^{\circ}$ & 12.47 & 2.8 & \multirow{5}{*}{3.257} & \multirow{5}{*}{.014} \\
\hline & $2^{\circ}$ & 11.04 & 3.25 & & \\
\hline & $3^{\circ}$ & 10.29 & 2.29 & & \\
\hline & $4^{\circ}$ & 11.72 & 4.06 & & \\
\hline & $5^{\circ}$ & 13.02 & 2.86 & & \\
\hline \multirow{5}{*}{ Reevaluación Positiva } & $1^{\circ}$ & 13.14 & 2.59 & \multirow{5}{*}{8.112} & \multirow{5}{*}{.000} \\
\hline & $2^{\circ}$ & 11.63 & 2.59 & & \\
\hline & $3^{\circ}$ & 10.58 & 2.06 & & \\
\hline & $4^{\circ}$ & 13.72 & 1.98 & & \\
\hline & $5^{\circ}$ & 12.83 & 2.36 & & \\
\hline \multirow{5}{*}{ Búsqueda de Apoyo } & $1^{\circ}$ & 9.33 & 3.19 & \multirow{5}{*}{6.020} & \multirow{5}{*}{.000} \\
\hline & $2^{\circ}$ & 8.31 & 3.18 & & \\
\hline & $3^{\circ}$ & 11.20 & 2.22 & & \\
\hline & $4^{\circ}$ & 10.47 & 3.23 & & \\
\hline & $5^{\circ}$ & 11.89 & 2.97 & & \\
\hline \multirow{5}{*}{ Evitación Cognitiva } & $1^{\circ}$ & 8.47 & 3.31 & \multirow{5}{*}{8.079} & \multirow{5}{*}{.000} \\
\hline & $2^{\circ}$ & 7.90 & 3.50 & & \\
\hline & $3^{\circ}$ & 10.72 & 2.64 & & \\
\hline & $4^{\circ}$ & 6.38 & 2.78 & & \\
\hline & $5^{\circ}$ & 7.02 & 3.21 & & \\
\hline \multirow{5}{*}{ Aceptación-Resignación } & $1^{\circ}$ & 6.81 & 3.26 & \multirow{5}{*}{8.925} & \multirow{5}{*}{.000} \\
\hline & $2^{\circ}$ & 5.04 & 3.13 & & \\
\hline & $3^{\circ}$ & 9.08 & 2.82 & & \\
\hline & $4^{\circ}$ & 4.69 & 3.91 & & \\
\hline & $5^{\circ}$ & 4.67 & 2.92 & & \\
\hline
\end{tabular}




\begin{tabular}{cccccc}
\hline & Año & $M$ & $D E$ & $F$ & $p$ \\
\hline & $1^{\circ}$ & 8.00 & 2.02 & & \\
Descarga Emocional & $2^{\circ}$ & 8.04 & 3.24 & & \\
& $3^{\circ}$ & 10.58 & 2.33 & 3.562 & .009 \\
& $4^{\circ}$ & 8.61 & 3.13 & & \\
\hline
\end{tabular}

Para profundizar en el análisis se realizó una prueba post hoc de Bonferroni. En la mayoría de las casos, los estudiantes de $3^{\circ}$ año obtuvieron los promedios extremos de las diferencias (ver Tabla 3).

\section{Tabla 3}

Análisis Post Hoc Bonferroni

\begin{tabular}{lcccc}
\hline & Curso & Curso & Dif de $M$ & $p$ \\
\hline Ansiedad & $3^{\circ}$ & $5^{\circ}$ & 8.9 & .000 \\
Análisis Lógico & $4^{\circ}$ & -8.4 & .001 \\
& $3^{\circ}$ & $5^{\circ}$ & -2.7 & .014 \\
Reevaluación Positiva & $1^{\circ}$ & $3^{\circ}$ & 2.5 & .003 \\
& $2^{\circ}$ & $4^{\circ}$ & -2.1 & .010 \\
Búsqueda de Apoyo & $3^{\circ}$ & $4^{\circ}$ & -3.1 & .000 \\
& $1^{\circ}$ & $5^{\circ}$ & -2.2 & .003 \\
Evitación Cognitiva & $5^{\circ}$ & -2.5 & .022 \\
& $2^{\circ}$ & $3^{\circ}$ & -2.9 & .014 \\
Aceptación-Resignación & $5^{\circ}$ & $3^{\circ}$ & -3.6 & .000 \\
& $2^{\circ}$ & $4^{\circ}$ & -2.8 & .025 \\
& $3^{\circ}$ & $5^{\circ}$ & 3.31 & .000 \\
Descarga Emocional & $2^{\circ}$ & $3^{\circ}$ & -4.03 & .000 \\
& $3^{\circ}$ & $4^{\circ}$ & 4.4 & .000 \\
& $1^{\circ}$ & $5^{\circ}$ & 4.4 & .000 \\
& $2^{\circ}$ & $3^{\circ}$ & -2.6 & .023 \\
& $3^{\circ}$ & $3^{\circ}$ & -2.5 & .024 \\
& & & 2.2 & .024 \\
\hline
\end{tabular}


Para comprobar la existencia de correlaciones entre la ansiedad ante exámenes y las estrategias de afrontamiento, se aplicó una prueba de correlación de Pearson, a partir de la cual se encontraron 5 correlaciones significativas débiles entre valores de ansiedad y estrategias de afrontamiento. Dos de ellas negativas: reevaluación positiva $(r=-.247 ; p<.01)$ y solución de problemas $(r=-.391 ; p<.001)$, $\mathrm{y}$ tres positivas: evitación cognitiva $(r=.355 ; p<.001)$; aceptaciónresignación $(r=.403 ; p<.001)$ y descarga emocional $(r=.232 ; p<.01)$ (ver Tabla 4).

\section{Tabla 4}

Prueba de Correlación de Pearson entre la Ansiedad y las Estrategias de Afrontamiento ante situación de examen en los estudiantes universitarios

Estrategias de Afrontamiento Ansiedad ante exámenes

\begin{tabular}{lcc}
\hline & $r$ & $p$ \\
\cline { 2 - 3 } Reevaluación Positiva & -.247 & $<.01$ \\
Solución de Problemas & -.391 & $<.001$ \\
Evitación Cognitiva & .355 & $<.001$ \\
Aceptación-Resignación & 403 & $<.001$ \\
Descarga Emocional & .232 & $<.01$ \\
\hline
\end{tabular}

Posteriormente, se realizó una prueba $t$ de Student para comparar si existían diferencias significativas entre el género de los estudiantes y las variables en estudio. Los resultados arrojaron diferencias significativas en las estrategias de Reevaluación Positiva $(t=2.441 ; p<.05)$ y Descarga Emocional $(t=2.277 ; p<.05)$. En el análisis del tamaño del efecto la diferencia observada en Reevaluación Positiva es media, mientras que en Descarga Emocional no es significativa (Cohen, 1988) (ver Tabla 5). 
Estrategias de afrontamiento y ansiedad ante exámenes en estudiantes universitarios / Casari et al.

\section{Tabla 5}

Prueba $t$ de Student según género (Femenino $n=130$; masculino $n=30$ )

\begin{tabular}{|c|c|c|c|c|c|c|}
\hline Nivel de Ansiedad & Género & $M$ & $D E$ & $t$ & $p$ & $d$ \\
\hline \multirow{2}{*}{$\begin{array}{l}\text { Reevaluación } \\
\text { Positiva }\end{array}$} & Femenino $(r$ & 12.8 & 2.33 & \multirow{2}{*}{2.441} & \multirow{2}{*}{$<.05$} & \multirow{2}{*}{.515} \\
\hline & Masculino $(\mathrm{n}=30)$ & 11.4 & 3.06 & & & \\
\hline \multirow{2}{*}{$\begin{array}{l}\text { Descarga } \\
\text { Emocional }\end{array}$} & Femenino ( $\mathrm{n}$ & 10.52 & 3.03 & \multirow{2}{*}{2.3} & \multirow{2}{*}{$<.05$} & \multirow{2}{*}{-.181} \\
\hline & Masculino $(\mathrm{n}=30)$ & 11.12 & 3.58 & & & \\
\hline
\end{tabular}

\section{Discusión}

En primer lugar, los resultados arrojaron que tanto hombres como mujeres presentaban un nivel bajo de ansiedad ante los exámenes. Si bien los niveles oscilan de baja a alta ansiedad, estos resultados difieren de los hallados en las investigaciones de Álvarez y colaboradores (2012), y Hernández (2005), en las cuales los alumnos evaluados manifestaron una alta ansiedad ante un examen en donde podrían necesitar ayuda especializada. No obstante, se consideran las diferencias interindividuales en el nivel de ansiedad como elevada, media o baja ansiedad ante exámenes; además de las diferencias intraindividuales en los niveles de ansiedad-estado, por la relación entre el rasgo individual de cada estudiante y los factores situacionales propios de cada evaluación (Castellanos et al., 2011).

La investigación se enmarca en el Modelo de la Reducción de la Eficiencia desarrollada por Gutiérrez Calvo y Avero (1995). Es decir, la preocupación se da sobre un mecanismo autorregulatorio que da señales de alerta que se generan cuando el estudiante compara los conocimientos que tiene con los conocimientos realmente necesarios para conseguir la aprobación del examen. Cuando en el estudiante estos no coinciden o superan los conocimientos necesarios, entonces crece la preocupación y el estudiante aumenta sus recursos extras como por ejemplo: dedicar más tiempo de estudio y de repaso, emplear 
alternativas de procesamiento cognitivo, planificar las actividades, buscar información que le ayude a lograr una mejor preparación, estudiar con un compañero/a, entre otras, con el objetivo de disminuir la preocupación evitando las posibilidades de fracaso (Piemontesi \& Heredia, 2009). Entonces, es importante diferenciar dos conceptos claves: la eficacia en el rendimiento y la eficiencia en el procesamiento, es decir, las estrategias de aprendizaje que implementan los alumnos en función de la preocupación que le genere el examen. De esta forma, en este modelo se explica por qué un estudiante que presenta alta ansiedad ante un examen puede tener un rendimiento eficaz, al igual que un estudiante con media o baja ansiedad, ya que la diferencia radica en la eficiencia del procesamiento, es decir, las estrategias que el estudiante implemente.

Por lo tanto, el hecho de que los estudiantes evaluados presentan en general un nivel bajo de ansiedad ante los exámenes, no implica un buen rendimiento, sino que su manera de procesar la información es más eficiente que los estudiantes de alta ansiedad, ya que estos implementan esfuerzos mayores con recursos extras para tener un buen rendimiento al procesar de manera menos eficiente. Resultados análogos se han encontrado en dos investigaciones. La primera, realizado por Furlan y colaboradores (2012), muestra que el uso de estrategias de repetición y memorización con un aprendizaje superficial de los contenidos se encuentra presente en el grupo de estudiantes con ansiedad alta; mientras que las estrategias de estudio reflexivo, como la elaboración y el pensamiento crítico, resultaron significativamente mayor en el grupo de baja ansiedad. El segundo estudio es de Álvarez y colaboradores (2012) en el cual los estudiantes evaluados manifiestan una ansiedad significativamente alta frente a los exámenes en respuestas cognitivas, fisiológicas y motoras; no obstante, el rendimiento académico no presentó relación con estos niveles de ansiedad, por lo cual se podría inferir que un estudiante con alta ansiedad ante un examen puede tener un rendimiento igualmente eficaz al estudiante con media o baja ansiedad. La diferencia radica en la eficiencia del procesamiento, ya que en el primero los esfuerzos son mayores para tener un buen rendimiento al 
procesar de manera menos eficiente (Piemontesi \& Heredia, 2009), a diferencia de las teorías del Modelo del Procesamiento de la Información y la Teoría del Déficit del Aprendizaje que postulan que los estudiantes con alta ansiedad obtienen puntuaciones más bajas en el examen al igual que los estudiantes con moderada o baja ansiedad, es decir, a mayor ansiedad, mejor rendimiento y viceversa (Miralles Muñoz, Cima Muñoz \& Jimenez Perianes, 2012).

Con respecto a las estrategias que utilizan con más frecuencia los estudiantes, los resultados arrojaron que son las de evitación conductuales, predominando un Afrontamiento basado en la evitación, es decir, que los comportamientos están orientados a distanciarse físicamente de la situación evaluativa. Asimismo, también se encuentran las estrategias de Descarga Emocional y la Búsqueda de Gratificaciones Alternativas. Cabe aclarar que esta última hace referencia a los intentos conductuales que emplean los estudiantes para realizar actividades nuevas y crear fuentes sustitutas de satisfacción, es decir, modificaciones de conducta en los estudiantes cuando se acerca la fecha de exámenes, como puede ser el aumento del consumo de cafeína, tabaco, sustancias psicoactivas, de comida, etc. (Martín Monzón, 2007). Con respecto a la Descarga Emocional, esta haría referencia a los intentos conductuales para reducir la tensión mediante la expresión de los sentimientos negativos cuando se acerca la fecha de exámenes, como puede ser que los estudiantes lloren, griten, tengan discusiones con sus pares o familia, expresiones de enojo, etc.

Este resultado difiere de tres investigaciones revisadas. En la primera (Sicre, 2013), se concluyó que la estrategia preferentemente utilizada por los estudiantes es el Afrontamiento por Aproximación Cognitiva, la cual comprende los esfuerzos cognitivos para entender y prepararse mentalmente para enfrentar un elemento generador de estrés y sus consecuencias. La segunda realizada por Rusińol y colaboradores (1993), halló que los estudiantes utilizan con mayor frecuencia la estrategia Reevaluación Positiva, siendo una estrategia cognitiva de acercamiento. Por último, González Cabanach, González Millán y Freire Rodríguez (2009) desarrollaron una investigación dónde se analizaron cuáles son 
las estrategias de afrontamiento a las que solían recurrir los estudiantes universitarios de ciencias de la salud. A diferencia de los resultados que se hallaron en la presente investigación, la estrategia de Búsqueda de Apoyo de Acercamiento Conductual fue la más frecuentemente utilizada, mientras que la Reevaluación Positiva de Acercamiento Cognitivo resultó ser la menos utilizada por los estudiantes.

Es preciso recordar que, para la mayoría de los autores, el afrontamiento orientado a la evitación, como lo es en la presente investigación, ayuda a los estudiantes a mantener un equilibrio emocional disminuyendo los niveles de tensión; no obstante, pueden ser estrategias menos adaptativas, como la Descarga Emocional y la Búsqueda de Gratificaciones Alternativas. Sin embargo, si estas estrategias se practican en exceso, como el uso de alcohol y drogas, o la excesiva ingesta de alimentos, pueden generar problemas en su salud. En ese sentido, se sugiere que para que resulte una respuesta efectiva a la situación de examen es necesario implementar las estrategias orientadas al problema, tales como la Resolución de Problemas, el Análisis Lógico, la Reevaluación Positiva y la Búsqueda de Apoyo, las cuales le permiten al estudiante dominar el estresor y no que la situación de examen los domine a ellos (Heredia et al., 2008).

Es necesario aclarar que la muestra arrojó que todas las estrategias de afrontamiento están presentes ante la situación de examen, lo que permite inferir que los estudiantes son más flexibles con mayor cantidad de recursos para la situación, mientras que utilizan con mayor predominancia las estrategias evitativas. Además, se debe tener en cuenta que la respuesta al estrés depende de la disposición personal para hacer frente a las demandas de la situación de examen, así como de la propia situación. Para esto, se recomienda recordar lo que postulan Piemontesi y Heredia (2009) en relación a las estrategias de afrontamiento, las cuales no deberían ser consideradas adaptativas o desadaptativas, sino que serían más bien funcionales y particulares dependiendo de cada estudiante.

A su vez, es pertinente considerar que las estrategias de afrontamiento que el estudiante emplea varían según las emociones pre- 
dominantes y las demandas conductuales de cada etapa de la situación evaluativa (anticipatoria, confrontación, espera y resultados) por lo que la misma estrategia implementada por distintos estudiantes puede ser más o menos adaptativa según la etapa en la cual se desenvuelve (Piemontesi \& Heredia, 2009). En la presente investigación, se consideró la situación de examen en general, no en una etapa específica, por lo que sería recomendable para próximas investigaciones estudiar las estrategias de afrontamiento según cada etapa de la situación evaluativa.

Con respecto a la relación entre los niveles de ansiedad y las estrategias de afrontamiento, se encontraron múltiples diferencias significativas entre estudiantes de distintos años académicos. Entre los estudiantes de $3^{\circ}$ y $5^{\circ}$ año se observan diferencias en Ansiedad, Análisis Lógico y Aceptación-Resignación. En cuanto a Reevaluación Positiva y Evitación Cognitiva se manifestaron entre los alumnos de $3^{\circ}$ y $4^{\circ}$ año. Entre los alumnos de $2^{\circ}$ y $5^{\circ}$ la diferencia se encontró en Búsqueda de Apoyo y por último Descarga Emocional entre los estudiantes de $1^{\circ}$ y $3^{\circ}$ año. Estos resultados se consideran dentro de lo esperable ya que nos muestra que, en su mayoría, los estudiantes de los primeros años utilizan estrategias de Evitación cognitivas (Evitación Cognitiva y Aceptación-Resignación) y conductuales (Descarga Emocional); mientras que los estudiantes de los últimos ańos utilizan con mayor frecuencia las estrategias de acercamiento cognitivas (Reevaluación Positiva y Análisis Lógico) y conductuales (Búsqueda de Apoyo) hacia la situación de examen. Además, los niveles de ansiedad van disminuyendo según los ańos. Por ello, se podría inferir que a medida que transcurren los ańos académicos, los estudiantes no solo van disminuyendo los niveles de ansiedad, sino que además modifican sus estrategias de afrontamiento al examen, lo cual les permite enfrentarlo de una manera más eficiente, con un mejor rendimiento. Estos resultados coinciden con el estudio realizado por Montańa (2011), en el cual los estudiantes de $5^{\circ}$ año utilizan la estrategia de Resolución de Problemas y de Acercamiento Conductual. A su vez, también coinciden con los resultados de Sicre (2013), en donde el afrontamiento por Aproximación Cognitiva se dio más elevado en los estudiantes de los últimos años. 
Asimismo, en cuanto a la relación entre los niveles de ansiedad y estrategias de afrontamiento, los resultados arrojaron que, a menores niveles de ansiedad, los estudiantes implementaban con mayor frecuencia estrategias de acercamiento como la Solución de Problemas (acercamiento conductual) y la Reevaluación Positiva (acercamiento cognitivo). Mientras que, a mayores niveles de ansiedad, se incrementaban las estrategias de evitación tanto cognitivas como la Aceptación-Resignación y Evitación Cognitiva, y conductuales como la Descarga Emocional. Por lo tanto, ello muestra una clara relación entre los niveles de ansiedad y afrontamiento, es decir, a mayores niveles de ansiedad, los estudiantes utilizan estrategias de evitación hacia el examen y, a menores niveles, utilizan estrategias de acercamiento al examen.

Estos resultados coinciden con dos investigaciones encontradas. En la primera, realizada por Castellanos y colaboradores (2011), se observó que los estudiantes de este estudio, a menor nivel de ansiedad, empleaban más la Resolución de Problemas, mientras que con altos niveles de ansiedad, utilizaban más la Evitación Cognitiva. A su vez, en segunda investigación (Montańa, 2011), se encontró que a mayores niveles de ansiedad se utilizaba más la estrategia de Escape-Evitación y Distanciamiento, en tanto que a menores niveles de ansiedad más se utiliza la Resolución de Problemas. Sin embargo, estos resultados difieren de lo hallado por Furlan y colaboradores (2012), quienes encontraron que a mayor nivel de Ansiedad, mayor Búsqueda de Apoyo.

En relación a las diferencias significativas de género en las estrategias de afrontamiento, los resultados arrojaron que la estrategia Reevaluación Positiva es utilizada con mayor frecuencia en mujeres; mientras que la estrategia Descarga Emocional es utilizada principalmente por los hombres. Por lo tanto, en las mujeres predomina una estrategia cognitiva de acercamiento; mientras que en los hombres predomina una estrategia conductual de evitación. Cabe aclarar en este punto que más de la mitad de la muestra pertenece al sexo femenino $(n=115)$ y que estos resultados contradicen distintas investigaciones previas.

Furlan (2013) sostiene que la prevalencia de ansiedad es mayor en las mujeres, lo que implica que en carreras donde hay más mujeres 
existe mayor prevalencia de niveles altos de ansiedad. Cabanach y colaboradores (2013) señalan que ambos sexos recurren a estrategias de acercamiento conductual: los hombres recurren en mayor medida a la Reevaluación Positiva, mientras que las mujeres optan fundamentalmente por la Búsqueda de Apoyo. Montaña (2011) refiere que las mujeres utilizan la estrategia de Resolución de Problemas y Búsqueda de Apoyo Social; mientras que los hombres no utilizan una estrategia con preferencia, pero sí presentan mayores niveles de emocionalidad, preocupación e interferencias. Por último, Rusiñol y colaboradores (1993) sostienen que el Distanciamiento es la estrategia de afrontamiento más utilizada por estudiantes hombres, sin embargo, coinciden con este estudio en el hecho de que observaron que las mujeres utilizan con mayor frecuencia que los hombres la Reevaluación Positiva.

Con respecto al último objetivo planteado, en relación a la cantidad de materias y el nivel de ansiedad, el resultado arrojó una tendencia negativa entre ambas, es decir, que se podría inferir que a mayor cantidad de materias rendidas, menores niveles de ansiedad, lo que indicaría que a medida que transcurren los años académicos, los estudiantes aprenden a manejar los niveles de ansiedad. Al no ser una correlación significativa, se puede pensar que en una muestra mayor, puedan darse resultados significativos entre estas dos variables, lo cual sugiere la posibilidad de evaluar este fenómeno en próximos estudios.

Es relevante mencionar que una de las principales limitaciones de esta investigación se encuentra vinculada a la muestra, ya que más del $80 \%$ de los participantes pertenece al sexo femenino, lo que podría restarle validez a los resultados. En razón a ello, se recomienda que en futuras investigaciones se trabaje con una población compuesta únicamente por mujeres, ya que la literatura previa sugiere niveles diferenciales de ansiedad entre los géneros (Furlan, 2013).

Relacionado a esto, otra limitación fue que se utilizó un muestreo no probabilístico, aunque se contó en promedio con 28 estudiantes por curso, por lo que se procuró lograr una representación homogénea de los estudiantes de cada curso. 
En síntesis, este estudio de carácter descriptivo y correlacional pudo lograr una aproximación al conocimiento de las estrategias de afrontamiento y al nivel de ansiedad que presentan los estudiantes de Psicología ante situación de examen. En futuras investigaciones podrían diseñarse programas que apunten a reducir la ansiedad ante exámenes, que cuenten con el uso de estos instrumentos que demostraron ser útiles para este fin, con el objetivo de intervenir con herramientas psicosociales en la mejora del rendimiento estudiantil y su adaptación al ámbito universitario (Sicre, 2013), tal como fue realizado en estudios previos (Medrano, 2011). El tránsito de un alumno por la universidad no tiene por qué ser una experiencia estresante, sino que dichas instituciones también deberían promover la salud estudiantil, como se sostiene desde los postulados de universidades saludables (Becerra Heraud, 2013).

\section{Referencias}

Álvarez, J., Aguilar J. M. \& Lorenzo, J. J. (2012). La Ansiedad ante los Exámenes en Estudiantes Universitarios: Relaciones con variables personales y académicas. Electronic Journal of Research in Educational Psychology, 10(26), 333-354. Recuperado de http:// www.investigacion-psicopedagogica.com/revista/articulos/26/ espannol/Art_26_702.pdf

Ávila-Toscano, J. H., Hoyos Pacheco, S. R., González, D. P. \& Cabrales Polo, A. (2011). Relación entre ansiedad ante los exámenes, tipos de prueba y rendimiento académico en estudiantes universitarios. Psicogente, 14(26), 255-268. Recuperado de http:// portal.unisimonbolivar.edu.co:82/rdigital/psicogente/index. $\mathrm{php} / \mathrm{psicogente}$

Becerra Heraud, S. (2013). Universidades Saludables: Una apuesta a una formación integral del estudiante. Revista de Psicología de la Pontificia Universidad Católica de Perú, 31(2), 287-314. Recuperado de http://revistas.pucp.edu.pe/index.php/psicologia/ article/view/7621/7870 
Cabanach, R. G., Fariña, F., Freire, C., González, P. \& Ferradás, M. (2013). Diferencias en el afrontamiento del estrés en estudiantes universitarios hombres y mujeres. European Journal of Education and Psychology, 6(1), 19-32. Recuperado de http://www.redalyc. org/pdf/1293/129327497002.pdf

Castellanos, M. T., Guarnizo, C. A. \& Salamanca, Y. (2011). Relación entre niveles de ansiedad y estrategias de afrontamiento en practicantes de psicología de una universidad colombiana. International Journal of Psychological Research, 4(1), 50-57. Recuperado de http://www.redalyc.org/pdf/2990/299022819007. pdf

Cohen, J. (1988). Statistical power analysis. Current directions in Psychological Science, 1(3), 98-101.

Furlan L., Perez E., Moyano M. \& Cassady J. (2010). Propiedades psicométricas y estandarización de la Escala de Ansiedad Cognitiva frente a los Exámenes a la población universitaria argentina. Evaluar, 10, 22-31. Recuperado de http://revistas.unc.edu.ar/ index.php/revaluar/article/view/457/426

Furlan, L., Sánchez-Rosas, J., Heredia, D., Piemontesi, S., Illbele, A. \& Martínez, M. (2012). Estrategias de aprendizaje y afrontamiento en estudiantes con elevada ansiedad frente a los exámenes. Anuario de Investigaciones de la Facultad de Psicología, 1(1), 130-141. Recuperado de www.revistas.unc.edu.ar/index.php/aifp.

Furlan, L. (2013). Una traba para los estudiantes: la ansiedad frente a los exámenes. Recuperado de http://www.unc.edu.ar/investigacion/ cienciaytecnologia/novedades-informacion-cyt/20132/unatraba-para-los-estudiantes-la-ansiedad-frente-a-los-examenes

García-Ros, R., Pérez-González, F., Pérez-Blasco, J. \& Natividad, L. A. (2012). Evaluación del estrés académico en estudiantes de nueva incorporación a la universidad. Revista Latinoamericana de Psicología, 44(2), 143-154. Recuperado de http://www.redalyc.org/ pdf/805/80524058011.pdf

González Cabanach, R., González Millán, P. \& Freire Rodríguez, C. (2009). El afrontamiento del estrés en estudiantes de ciencias 
de la salud. Diferencias entre hombres y mujeres. Aula abierta, 37(2), 3-10. Recuperado de http://dialnet.unirioja.es/servlet/ articulo? codigo $=3088531$

Gutiérrez Calvo, M. \& Avero, P. (1995). Ansiedad, estrategias auxiliares y comprensión lectora: Déficit de procesamiento versus falta de confianza. Psicothema, 7(3), 569-578. Recuperado de http:// www.psicothema.com/pdf/1003.pdf

Heredia, D., Piemontesi, S., Furlan, L. \& Volker, H. (2008). Adaptación del Inventario Alemán de Ansiedad frente a los Exámenes: GTAI-A. Evaluar, 8, 46-60. Recuperado de http://revistas.unc. edu.ar/index.php/revaluar/article/view/504/460

Hernández, J. M. (2005). Ansiedad ante los exámenes: una evaluación de sus manifestaciones en los estudiantes universitarios españoles. Education, 13-18.

Martín Monzón, I. M. (2007). Estrés académico en estudiantes universitarios. Apuntes de Psicología, 25(1), 87-99. Recuperado de http://www.apuntesdepsicologia.es/index.php/revista/article/ viewFile/117/119

Medrano, L. (2011). Evaluación de la eficacia de un programa de entrenamiento para ingresantes universitarios: Su impacto en la ansiedad ante exámenes. En L. Medrano (Ed.), Prácticas en salud mental basadas en la evidencia (pp. 116-126.). Córdoba: Universidad Nacional de Córdoba.

Mikulic, I. M. \& Crespi, M. C. (2008). Adaptación y validación del Inventario de Respuestas de Afrontamiento de Moos (CRI-A) para adultos. Anuario de Investigaciones de la Facultad de Psicologia de la Universidad de Buenos Aires, Vol. XV, 305-312.

Miralles Muñoz, F., Cima Muñoz, A. M. \& Jimenez Perianes, A. (2012, marzo). Ansiedad en los procesos de evaluación: herramientas para su Manejo. Escuela y Psicopatología, 2, 26-77. Recuperado de http://www.fernandomiralles.es/ansiedadexamenes.pdf

Montaña, L. A. (2011). Ansiedad en situación de examen y estrategias de afrontamiento en alumnos universitarios de $1^{\circ}$ y $5^{\circ}$ año. Tesis de Licenciatura para optar el título de Licenciado en Psicología, 
Facultad de Psicología y Relaciones Humanas, Universidad Abierta Interamericana., Buenos Aires, Argentina.

Piemontesi, S. E. \& Heredia, E. D. (2009). Afrontamiento ante exámenes: Desarrollos de los principales modelos teóricos para su definición y medición. Anales de Psicología, 25(1), 102-111. Recuperado de http://revistas.um.es/analesps/article/view/71551

Piemontesi, S. E., Heredia, E. D., Furlan, L. A., Sánchez-Rosas, J. \& Martínez, M. (2012). Ansiedad ante los exámenes y estilos de afrontamiento ante el estrés académico en estudiantes universitarios. Anales de Psicología, 28(1), 89-96. Recuperado de http:// revistas.um.es/analesps/article/view/140562

Polo, A., Hernández, J. M. \& Pozo, C. (1996). Evaluación del Estrés Académico en Estudiantes Universitarios. Ansiedad y Estrés, 2(2-3), 159-172. Recuperado de http://www.unileon.es/estudiantes/ atencion_universitario/articulo.pdf

Ricoy, M. C. \& Fernandez-Rodriguez, J. (2013). La percepción que tienen los estudiantes universitarios sobre la evaluación: un estudio de caso. Educación XX1, 16(2), 321-342. Recuperado de http://www.redalyc.org/pdf/706/70626451006.pdf

Rusińol, J., Gómez, J., De Pablo, J. \& Valdés, M. (1993). Relación entre estrategias de afrontamiento y la ansiedad ante la situación de examen. Revista de Psicología General y Aplicada, 46(1), 71-74.

Sicre, E. (2013). Estrés académico y Estrategias de afrontamiento frente a exámenes en estudiantes universitarios. Tesis de Licenciatura para optar el título de Licenciado en Psicología, Facultad de Humanidades y Ciencias de la Educación, Universidad Católica Argentina, Mendoza, Argentina.

Recibido: 28 de marzo, 2014 Aceptado: 30 de abril, 2014 